\title{
Type 2 diabetes and lifetime alcohol use disorder interact to predict poor endothelial function in postmenopausal women.
}

Omar Braizat ${ }^{1}$, Sharif Adwan ${ }^{1}$, Wadah Akroush ${ }^{1}$, Ahmad Mesmar ${ }^{1}$, Julie Wagner ${ }^{2}$

University of Jordan Hospital ${ }^{1}$ University of Connecticut Health Center ${ }^{2}$

\section{Background and Aims}

Diabetes is the only disorder in which women's risk for heart disease exceeds that of men's '. Flow mediated dilation (FMD) is a non-invasive measure of endothelial function and a strong predictor of cardiovascular mortality ${ }^{2}$. Type 2 diabetes (T2DM) ${ }^{3}$ and lifetime history of alcohol use disorder (LAUD) ${ }^{4}$ are each associated with decrements in FMD. Virtually nothing is known about the association between AUD and FMD in the setting of T2DM. This study investigated associations among T2DM, AUD, and FMD in women.
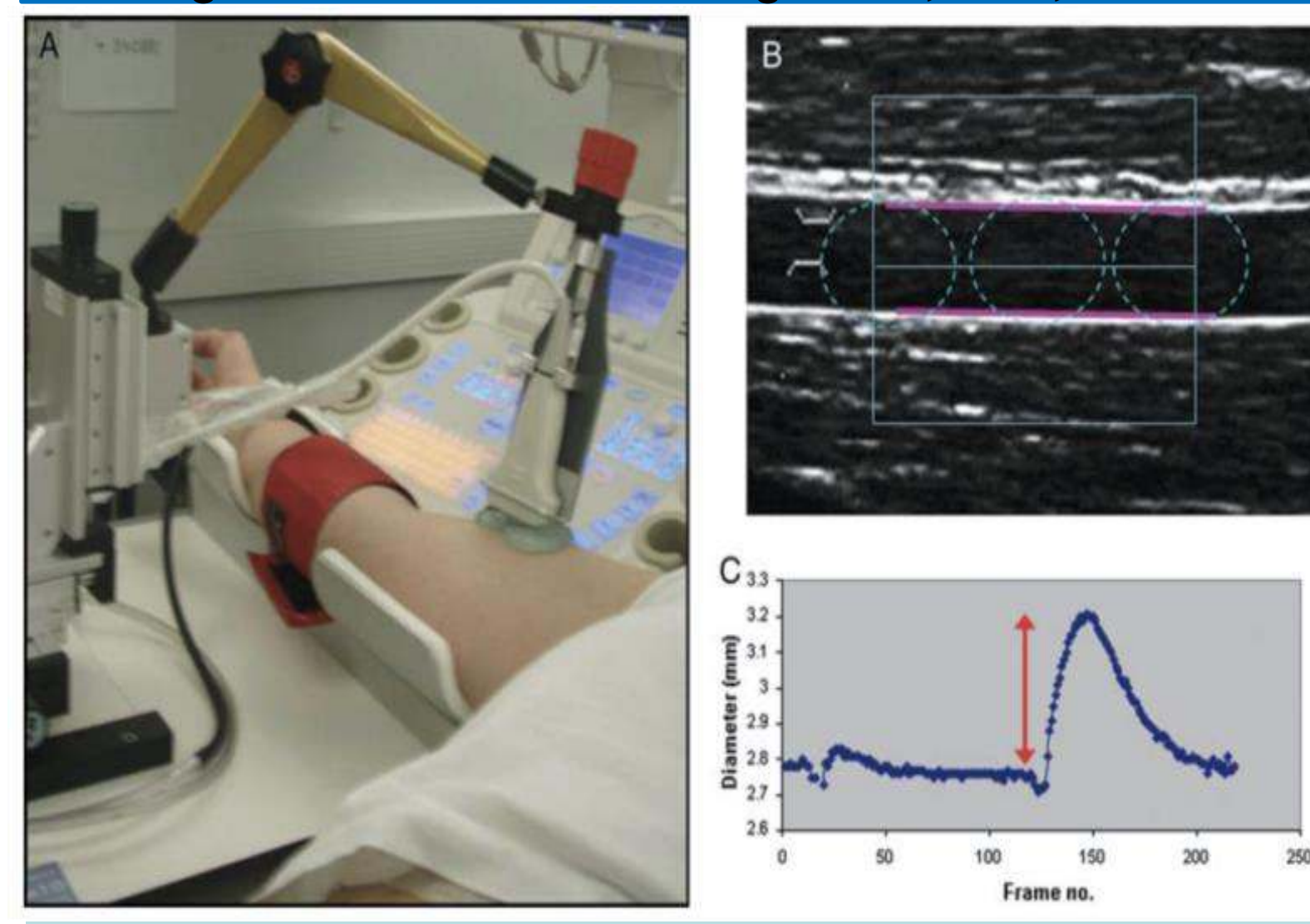

Methods

\section{Subjects}

Recruitment: Women were recruited from Connecticut, USA using state employee paycheck inserts, newspaper advertisements and list serves.

Inclusion criteria: Female; naturally postmenopausal. Women with T2DM were oversampled. Exclusion criteria: Current smoking, current substance use disorder, current mood disorder, known cardiovascular disease, and double oopherectomy.

\section{Measures and Procedures}

LAUD was assessed with Structured Clinical Interview for DSM-IV by doctoral level psychologists.

Endothelial function was measured per established guidelines ${ }^{5}$ for FMD. A longitudinal, diastolic gated ultrasound image of the brachial artery was captured, followed by occlusion of the artery distal to the study site with an inflated blood pressure cuff for 5 minutes and then released. Post-inflation image of the same artery segment was captured. Finally, after 20-minutes rest, sublingual nitroglycerin was administered and endothelium-independent dilation was assessed.

Ultrasound images were analyzed offline by two blinded, trained technicians using Vascular Research Tools software (Medical Imaging Applications, USA). FMD is calculated as percent change from baseline; higher percent dilation indicates better endothelial function.

Covariates included age, ethnicity, history of smoking, family history of heart disease, low-density lipoproteins, daily aspirin use and lifetime history of major depressive disorder.

A 2 (T2DM yes vs no) X 2 (LAUD yes vs no) analysis of variance was conducted in SPSS v20.

\section{UCDNN HE A LT H}

\section{Results}

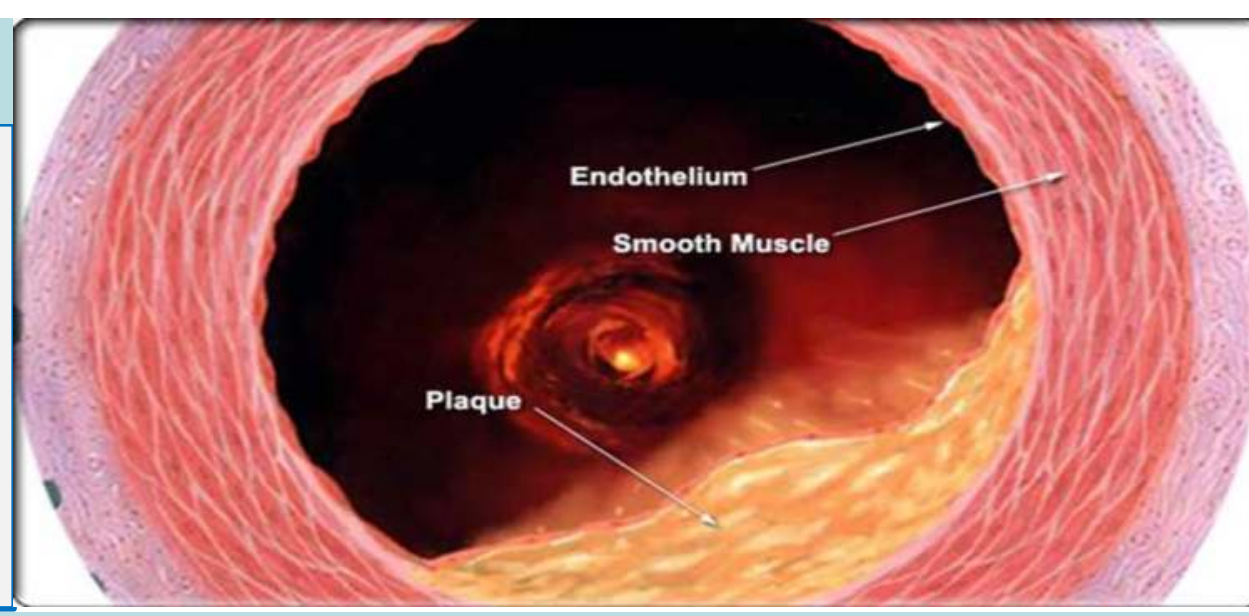

- 208 naturally postmenopausal women aged 45- 80 years were included; about half (49\%) had T2DM and one-quarter $(23 \%)$ had LAUD. Mean age was 61.6 years (SD=8.0), 84\% were White, and the majority were married (56\%).

- There was a significant interaction between T2DM and LAUD on FMD ( $p=.04)$. LAUD was associated with greater decrements in FMD among women with T2DM compared to non-diabetic women. The interaction remained significant after controlling for multiple potential confounds $(p=.03)$. Women with +DM+LAUD showed paradoxical vasoconstriction, indicative of severe endothelial dysfunction.

- There was a main effect for T2DM on FMD; -DM mean $=3.34 \%$ (SD=7.76) vs +DM mean=2.55\% (SD=7.69), $p=.04$, and for LAUD on FMD; -AUD mean=3.32\% (SD=7.58) vs +AUD mean $=1.35 \%$ $(\mathrm{SD}=8.19), \mathrm{p}=.04$.

- There were no differences for endothelium independent dilation with nitroglycerine, $p>.27$.

Flow Mediated Dilatation (FMD) depending

on Lifetime Alcohol Use disorder (LAUD) and

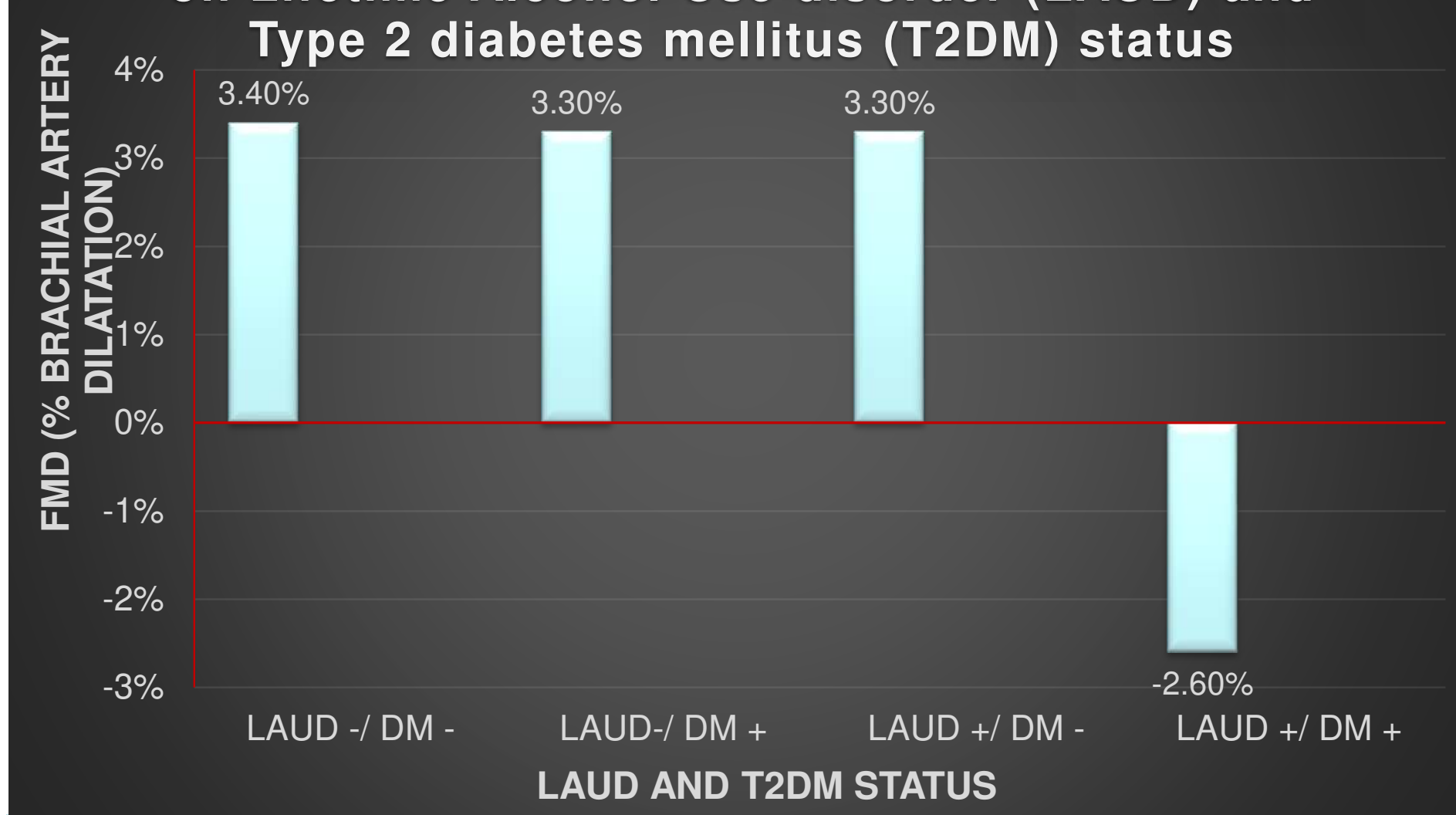

Discussion

- Lifetime history of fully remitted AUD has a more deleterious association with FMD in women with T2DM compared to their non-diabetic counterparts.

- Very small group differences in FMD are predictive of future cardiac events ${ }^{2}$. Thus, the differences observed in this study have clear clinical significance.

- Endothelial dysfunction is one mechanism through which LAUD confers risk for poor cardiovascular outcomes.

Limitations include a small sample, few minority women which precluded examination of ethnic differences, a low percentage of participants reporting LAUD, and potential biases in recall and reporting of LAUD.

- Efforts to prevent or reduce exposure to LAUD may improve the cardiovascular profile of postmenopausal women with T2DM.

\section{References}

1. Lyon A et al. Sex-specific differential in risk of diabetes-related macrovascular outcomes. Curr Diab Rep 2015;15 (11)85. 2. Gokce $N$ et al. Predictive value of noninvasively determined endothelial dysfunction for long-term cardiovascular events in patients with peripheral vascular disease. J Am Coll Cardiol 2003;41:1769-1775.

3. Tabit CE, et al. Endothelial dysfunction in diabetes mellitus: Molecular mechanisms and clinical implications. Rev Endocr Metab Disord;2010: 11:61-74.

4. Goslawski M, et al. Binge drinking impairs vascular function in young adults. J Am Coll Cardiol 2013;62:201-207. 5. Deanfield $\mathrm{J}$ et al. Guidelines for the ultrasound assessment of endothelial-dependent flow-mediated vasodilation of the 